\title{
Hodge equations with change of type
}

\author{
Thomas H. Otway* \\ Department of Mathematics and Department of Physics, \\ Yeshiva University, 500 W 185th Street, \\ New York, New York 10033
}

\begin{abstract}
A geometric interpretation is given for certain elliptic-hyperbolic systems in the plane. Among several examples, one which reduces in the elliptic region to the equations for harmonic 1-forms on the projective disc is studied in detail. A boundary-value problem for this example is formulated and is shown to possess weak solutions. MSC2000: 35M10, 58J99.
\end{abstract}

\section{Introduction}

Harmonic forms $\alpha$ on a Riemannian manifold satisfy the Hodge equations

$$
\delta \alpha=d \alpha=0,
$$

where $d$ is the exterior derivative and $\delta$ its adjoint. If we write these equations on a singular surface, it may happen that they can be rewritten as a system of mixed type in $\mathbb{R}^{2}$ in which the parabolic curve lies along the singularity of the surface. This yields a geometric interpretation of certain elliptic-hyperbolic systems in the plane.

For example, consider Beltrami's hyperbolic model [B1] for the projective disc $\mathbb{P}^{2}$. The metric tensor in this model is the matrix

$$
G_{i j}=\frac{1}{\left(1-x^{2}-y^{2}\right)^{2}}\left[\begin{array}{cc}
1-y^{2} & x y \\
x y & 1-x^{2}
\end{array}\right] .
$$

*email: otway@ymail.yu.edu 
Equations (1) for 1 -forms $u=\left(u_{1}, u_{2}\right)$ on $\mathbb{P}^{2}$ have the local form

$$
\begin{gathered}
|G|^{-1 / 2} \partial_{i}\left(G^{i j} \sqrt{|G|} u_{j}\right)=0 \\
\partial_{i} u_{j} d x^{i} \wedge d x^{j}=0 .
\end{gathered}
$$

The matrix

$$
G^{i j}=\left(1-x^{2}-y^{2}\right)\left[\begin{array}{cc}
1-x^{2} & -x y \\
-x y & 1-y^{2}
\end{array}\right]
$$

becomes indefinite, and the determinant

$$
G=\frac{1}{1-x^{2}-y^{2}}
$$

becomes singular, on the line at infinity of the model, which corresponds to the circle $x^{2}+y^{2}=1$.

But the equations can be redefined so that the metric singularity on the unit circle in $\mathbb{P}^{2}$ is replaced by a change of type on the unit circle in $\mathbb{R}^{2}$. Writing out eq. (2) in coordinates, we obtain

$$
\begin{gathered}
\left(1-x^{2}-y^{2}\right)\left\{\left[\left(1-x^{2}\right) u_{1}\right]_{x}-\left(x y u_{1}\right)_{y}-\left(x y u_{2}\right)_{x}\right. \\
\left.+\left[\left(1-y^{2}\right) u_{2}\right]_{y}-\left(x u_{1}+y u_{2}\right)\right\}=0 .
\end{gathered}
$$

Equation (3) implies that

$$
\left(x y u_{1}\right)_{y}+\left(x y u_{2}\right)_{x}=2 x y u_{1 y} .
$$

Outside the unit circle the projective disc model no longer applies, but eqs. (4), (5) are well defined and possess wave-like solutions in which disturbances propagate along null geodesics of the distance element

$$
d s^{2}=\frac{\left(1-y^{2}\right) d x^{2}+2 x y d x d y+\left(1-x^{2}\right) d y^{2}}{\left(1-x^{2}-y^{2}\right)^{2}} .
$$

In order for a 1 -form $u$ to satisfy (4), (5), it is sufficient for $u$ to satisfy a system of first-order equations on $\mathbb{R}^{2}$ having the form

$$
L u=g,
$$

where

$$
L=\left(L_{1}, L_{2}\right), g=\left(g_{1}, g_{2}\right),
$$




$$
\begin{gathered}
u=\left(u_{1}(x, y), u_{2}(x, y)\right),(x, y) \in \Omega \subset \subset \mathbb{R}^{2} \\
(L u)_{1}=\left[\left(1-x^{2}\right) u_{1}\right]_{x}-2 x y u_{1 y}+\left[\left(1-y^{2}\right) u_{2}\right]_{y}-2\left(x u_{1}+y u_{2}\right),
\end{gathered}
$$

and

$$
(L u)_{2}=\left(1-y^{2}\right)\left(u_{1 y}-u_{2 x}\right)
$$

If $g_{2}=0$ and $y^{2} \neq 1$, then (8) is equivalent to the condition that the 1 -form $u$ be closed under exterior differentiation.

The second-order terms of eqs. (7), (8) can be written in the form

$$
L[u]=A u_{x}+B u_{y}
$$

where

$$
A=\left[\begin{array}{cc}
1-x^{2} & 0 \\
0 & -\left(1-y^{2}\right)
\end{array}\right]
$$

and

$$
B=\left[\begin{array}{cc}
-2 x y & 1-y^{2} \\
1-y^{2} & 0
\end{array}\right] \text {. }
$$

If $y^{2} \neq 1$, the characteristic equation

$$
|A-\lambda B|=-\left(1-y^{2}\right)\left[\left(1-y^{2}\right) \lambda^{2}+2 x y \lambda+\left(1-x^{2}\right)\right]
$$

possesses two real roots $\lambda_{1}, \lambda_{2}$ on $\Omega$ precisely when $x^{2}+y^{2}>1$. Thus the system (6)-(8) is elliptic in the intersection of $\Omega$ with the open unit disc centered at $(0,0)$ and hyperbolic in the intersection of $\Omega$ with the complement of the closure of this disc. The boundary of the unit disc, along which this change in type occurs, is the line at infinity in $\mathbb{P}^{2}$ and a line singularity of the tensor $G_{i j}$.

L. K. Hua used variable separation, Poisson kernel, and D'Alembert methods to solve boundary-value problems for a scalar equation which resembles the system (6)-(8) $[\mathbf{H}]$. Precisely, the scalar equation studied by Hua consists of the conserved quantities in an equation which can be obtained from (7) and ( 8 ) by choosing $u_{1}=u_{x}, u_{2}=u_{y}$, and $g_{1}=g_{2}=0$. Inside the unit disc, these choices correspond to replacing the Hodge operator on 1-forms with the Laplace-Beltrami operator on scalars. By analogy with the approach in $[\mathbf{H}]$, we replace eq. (7) in the sequel by the equation

$$
(L u)_{1}=\left[\left(1-x^{2}\right) u_{1}\right]_{x}-2 x y u_{1 y}+\left[\left(1-y^{2}\right) u_{2}\right]_{y} .
$$

The system (6)-(8) with this replacement has the same hybrid qualities as the original version and the same boundary behavior, but has the property that $L^{*}=-L$ (c.f. Proposition 1). Subsequent references to the system (6)-(8) refer to the equations (6), (7'), and (8). 


\subsection{Other systems of mixed type}

The analogy between eqs. (2), (3) and the system (6)-(8) can be extended to other equations of mixed type, although generally these systems will have less interesting geometry than the projective disc. Perhaps the simplest case is a metric on which the Hodge equations reduce to Laplace's equation on one side of the metric singularity and to the wave equation on the other side. This leads to a system of the form

$$
\begin{gathered}
u_{1 x}+\operatorname{sgn}(y) u_{2 y}=0, \\
u_{1 y}-u_{2 x}=0,
\end{gathered}
$$

which corresponds in the case $u_{1}=u_{x}, u_{2}=u_{y}$ to the Lavrent'ev-Bitsadze equation. Moreover, the system introduced by Morawetz $[\mathbf{M}]$ as a vehicle for studying the Chaplygin equations is of a broadly similar form, as is the system studied in $[\mathbf{Y}]$.

The geometry of eqs. (6)-(8) is in some sense dual to that of a well known transform of the velocity potential for transonic flow in the hodograph plane. Denote by $\left(u_{1}(x, y), u_{2}(x, y)\right)$ the velocity components of a steady flow expressed in coordinates $(x, y)$. The hodograph transformation introduces $u_{1}$, $u_{2}$ as independent coordinates. The continuity equations for the velocity potential can now be written in the linear form $([\mathbf{B 2}]$, eq. (3.6))

$$
\begin{gathered}
\left(c^{2}-u_{1}^{2}\right) y_{u_{2}}+u_{1} u_{2}\left[x_{u_{2}}+y_{u_{1}}\right]+\left(c^{2}-u_{2}^{2}\right) x_{u_{1}}=0 \\
x_{u_{2}}-y_{u_{1}}=0
\end{gathered}
$$

Here

$$
c^{2}=1-\frac{\gamma_{a}-1}{2}\left(u_{1}^{2}+u_{2}^{2}\right)
$$

where $\gamma_{a}>1$ is the adiabatic constant of the medium. This system corresponds to our system where the parabolic curve is a circle of radius $\sqrt{2 /\left(\gamma_{a}+1\right)}$ centered at the point $u_{1}=0, u_{2}=0$ and the metric tensor in eqs. (2), (3) is the matrix

$$
\widetilde{G}_{i j}=\frac{1}{c^{2}\left(c^{2}-u_{2}^{2}-u_{1}^{2}\right)}\left[\begin{array}{cc}
c^{2}-u_{1}^{2} & -u_{1} u_{2} \\
-u_{1} u_{2} & c^{2}-u_{2}^{2}
\end{array}\right] .
$$

Consider for simplicity the lower limit of the range of values for $\gamma_{a}$, in which $c^{2}$ is approximately normalized. In this artificially simple case, the change of 
type occurs on the boundary of the unit circle and the continuity equations in the hodograph plane reduce to a replacement the metric tensor $G_{i j}\left(u_{1}, u_{2}\right)$ for eq. (2) by the tensor $\left(1-u_{1}^{2}-u_{2}^{2}\right)^{-2} G^{i j}\left(u_{1}, u_{2}\right)$ (again ignoring lowerorder terms). We obtain a second-order scalar equation if we introduce the "Legendre transform" $\chi\left(u_{1}, u_{2}\right)$ satisfying

$$
x=\chi_{u_{1}}, y=\chi_{u_{2}}
$$

(c.f. eq. (3.8) of [B2]). The characteristic curves of the resulting equation are relatively complicated, as they are given by a family of epicycloids which intersect the parabolic curve in a family of cusps. This leads to complicated boundary-value problems for the equation. By contrast, the characteristic curves corresponding to the "dual" system (6)-(8) are exceedingly simple, as they are given by the set of all tangent lines to the unit disc. This leads in our case to relatively simple boundary-value problems. How much can be said a priori about relations between solutions of the two sets of boundary-value problems is not immediately clear, however.

An alternative to considering the functions $u_{1}, u_{2}$ to be components of a 1 -form in $\mathbb{R}^{2}$ is to treat them as components of a function in $\mathbb{C}$. This is a standard approach in which, for example, the continuity equations in the hodograph plane are associated with a generalized Cauchy-Riemann operator. Among its many advantages, this approach has the disadvantage of giving special emphasis to dimension 2 and to the conformal group (or to quasiconformal mappings in the quasilinear case). In fact, the natural invariance group for eqs. (6)-(8) is the projective group rather than the conformal group, a circumstance which has some interesting consequences. For instance, whereas there are many conic sections in $\mathbb{R}^{2}$, the unit circle is one of only a few conic sections in the real projective plane; so the parabolic degeneracy at the point at infinity in the projective metric corresponds under projective mappings to a variety of parabolic curves in a euclidean metric (c.f. [VY], Sec. V.86; [H], p. 633).

\section{The boundary-value problem}

The Dirichlet problem for the system (6)-(8) involves prescribing the values of the 1 -form $u_{1} d x+u_{2} d y$ on the boundary of a domain of $\mathbb{R}^{2}$.

Denote by $\Omega$ the region bounded by the characteristic line $\Gamma$ tangent to the unit disc at the point $(1,0)$ and a smooth curve $C$ which intersects $\Gamma$ at 
exactly two points on the interval $-\sqrt{1-\delta}<y<-\nu$, where $\delta \in(0,1)$ and $\nu \in[0,1)$. Assume that $C$ lies entirely in the fourth quadrant and that it is bounded on the left by the line $x=\sqrt{1-\mu}$ for $\mu \in(0,1)$, on the right by the line $\Gamma$, above by the line $y=-\nu$, and below by the line $y=-\sqrt{1-\delta}$. Orient $\partial \Omega$ in the counterclockwise direction. We assume that, with this orientation, the line element $d y$ is nonpositive on $C$. (Because the system (6)-(8) is invariant under reflection about the origin, obvious modifications of these conditions will define an analogous boundary-value problem in the second quadrant.)

In Secs. 3 and 4 we show the existence and uniqueness of weak solutions to (6)-(8) which satisfy the boundary condition

$$
u_{1} \frac{d x}{d s}+u_{2} \frac{d y}{d s}=0
$$

where $s$ denotes arc length, on an appropriate boundary segment. The proof is based on methods introduced in $[\mathbf{M}]$ for boundary-value problems in the Chaplygin model.

Denote by $U$ the vector space consisting of all pairs of measurable functions $u=\left(u_{1}, u_{2}\right)$ for which the weighted $L^{2}$ norm

$$
\|u\|_{*}=\left[\iint_{\Omega} y^{2 \kappa}\left(u_{1}^{2}+u_{2}^{2}\right) d x d y\right]^{1 / 2}
$$

is finite, where

$$
\kappa=\frac{\mu+\varepsilon}{4(1-\mu)}
$$

for some $\varepsilon>0$. As this norm induces the weighted inner product

$$
(u, w)_{*}=\iint_{\Omega} y^{2 \kappa}\left(u_{1} w_{1}+u_{2} w_{2}\right) d x d y
$$

the space $U$ is a Hilbert space.

Denote by $W$ the Hilbert space defined by pairs of functions $w=\left(w_{1}, w_{2}\right)$, vanishing at the origin, having continuous derivatives, and satisfying

$$
w_{1} d x+w_{2} d y=0
$$

on $\gamma=\partial \Omega \cap \Gamma$ and

$$
w_{1}=0
$$


on $C$ for which the integral

$$
\iint_{\Omega} y^{-2 \kappa}\left[(L w)_{1}^{2}+(L w)_{2}^{2}\right] d x d y
$$

is finite.

Definition. We say that $u$ is a weak solution of the system (6)-(8) with boundary condition (9) if $u \in U$ and for every $w \in W$,

$$
(w, g)=-(L w, u)
$$

where

$$
(w, g)=\iint_{\Omega}\left(w_{1} g_{1}+w_{2} g_{2}\right) d x d y
$$

Proposition 1 Any continuously differentiable weak solution of the boundaryvalue problem (6)-(8), (9) is a classical solution.

Proof. In the interest of generality, we prove the proposition by an argument that applies to any smooth domain having a characteristic line segment on the boundary; we do not use any of the special properties of the line $x=1$ or of the fourth quadrant.

$$
\begin{aligned}
(L w)_{1} u_{1}= & {\left[\left(1-x^{2}\right) w_{1}\right]_{x} u_{1}-2 x y w_{1 y} u_{1}+\left[\left(1-y^{2}\right) w_{2}\right]_{y} u_{1} } \\
& =\left[\left(1-x^{2}\right) w_{1} u_{1}\right]_{x}-\left(1-x^{2}\right) w_{1} u_{1 x} \\
& -\left[2 x y w_{1} u_{1}\right]_{y}+2 x w_{1} u_{1}+2 x y w_{1} u_{1 y} \\
& +\left[\left(1-y^{2}\right) w_{2} u_{1}\right]_{y}-\left(1-y^{2}\right) w_{2} u_{1 y}
\end{aligned}
$$

and

$$
\begin{gathered}
(L w)_{2} u_{2}=\left(1-y^{2}\right)\left(w_{1 y}-w_{2 x}\right) u_{2}= \\
{\left[\left(1-y^{2}\right) w_{1} u_{2}\right]_{y}+2 y w_{1} u_{2}-\left(1-y^{2}\right) w_{1} u_{2 y}} \\
-\left[\left(1-y^{2}\right) w_{2} u_{2}\right]_{x}+\left(1-y^{2}\right) w_{2} u_{2 x} .
\end{gathered}
$$

Application of Green's Theorem to $(L w)_{1} u_{1}$ on the line segment $\gamma$ yields

$$
I_{1} \equiv \int_{\gamma}\left(1-x^{2}\right) w_{1} u_{1} d y+\left[2 x y w_{1} u_{1}-\left(1-y^{2}\right) w_{2} u_{1}\right] d x=
$$




$$
\int_{\gamma}\left[2 x y w_{1} u_{1}+\left(1-x^{2}\right) w_{1} u_{1}\left(\frac{d y}{d x}\right)-\left(1-y^{2}\right) w_{2} u_{1}\right] d x .
$$

From the boundary condition for elements of $W$ we obtain

$$
I_{1}=\int_{\gamma}\left\{2 x y w_{1} u_{1}-\left[\left(1-x^{2}\right)\left(\frac{d y}{d x}\right)^{2}+\left(1-y^{2}\right)\right] w_{2} u_{1}\right\} d x .
$$

On the characteristic curves,

$$
\left(1-y^{2}\right) d x^{2}+2 x y d x d y+\left(1-x^{2}\right) d y^{2}=0,
$$

so

$$
\left(1-x^{2}\right) \frac{d y^{2}}{d x^{2}}=-\left(1-y^{2}\right)-2 x y \frac{d y}{d x} .
$$

Substituting (12) into (11) yields

$$
\begin{gathered}
I_{1}=\int_{\gamma}\left\{2 x y w_{1} u_{1}-\left[-\left(1-y^{2}\right)-2 x y \frac{d y}{d x}+\left(1-y^{2}\right)\right] w_{2} u_{1}\right\} d x \\
=\int_{\gamma} 2 x y u_{1}\left(w_{1}+w_{2} \frac{d y}{d x}\right) d x=0 .
\end{gathered}
$$

Applying Green's Theorem to $(L w)_{2} u_{2}$ yields a boundary integral of the form

$$
I_{2} \equiv-\int_{\gamma}\left(1-y^{2}\right) u_{2}\left(w_{1} d x+w_{2} d y\right)=0 .
$$

Because $w_{1}$ vanishes on $C$, the result of applying Green's Theorem to $(L w, u)$ on $C$ is a boundary integral of the form

$$
\begin{aligned}
I_{3} \equiv & -\int_{C}\left(1-y^{2}\right) w_{2} u_{1} d x+\left(1-y^{2}\right) u_{2} w_{2} d y \\
& =-\int_{C}\left(1-y^{2}\right) w_{2}\left(u_{1} d x+u_{2} d y\right) .
\end{aligned}
$$

We obtain

$$
(L w, u)=\left(w, L^{*} u\right)-\int_{C}\left(1-y^{2}\right) w_{2}\left(u_{1} d x+u_{2} d y\right),
$$

where

$$
\left(w, L^{*} u\right)=
$$




$$
\begin{gathered}
-\iint_{\Omega}\left[\left(1-x^{2}\right) w_{1} u_{1 x}-2 x w_{1} u_{1}-2 x y w_{1} u_{1 y}+\left(1-y^{2}\right) w_{2} u_{1 y}\right] d x d y \\
+\iint_{\Omega}\left[2 y w_{1} u_{2}-\left(1-y^{2}\right) w_{1} u_{2 y}+\left(1-y^{2}\right) w_{2} u_{2 x}\right] d x d y= \\
-\iint_{\Omega}\left\{\left[\left(1-x^{2}\right) u_{1}\right]_{x}-2 x y u_{1 y}+\left[\left(1-y^{2}\right) u_{2}\right]_{y}\right\} w_{1} d x d y \\
-\iint_{\Omega}\left(1-y^{2}\right)\left[w_{2} u_{1 y}-w_{2} u_{2 x}\right] d x d y \\
=-\iint_{\Omega}\left[w_{1}(L u)_{1}+w_{2}(L u)_{2}\right] d x d y=-(w, L u)
\end{gathered}
$$

Combining eqs. (10) and (13) yields

$$
\begin{gathered}
(w, g)=-(L w, u)= \\
(w, L u)+\int_{C}\left(1-y^{2}\right) w_{2}\left(u_{1} d x+u_{2} d y\right) .
\end{gathered}
$$

Because $w$ is arbitrary in $W$ and $u$ is continuously differentiable, we conclude that (9) is satisfied on $C$ and $L u=g$, which completes the proof.

In Secs. 3 and 4 we prove:

Theorem 2 There exists a unique weak solution of the boundary-value problem (6)-(8), (9) on $\Omega$.

In proving Theorem 2 it will be necessary to introduce one additional Hilbert space. This is the space $H$ of pairs of measurable functions $h=$ $\left(h_{1}, h_{2}\right)$ for which the norm

$$
\|h\|^{*}=\left[\iint_{\Omega} y^{-2 \kappa}\left(h_{1}^{2}+h_{2}^{2}\right) d x d y\right]^{1 / 2}
$$

is finite. This norm is induced by the inner product

$$
(s, t)^{*}=\iint_{\Omega} y^{-2 \kappa}\left(s_{1} t_{1}+s_{2} t_{2}\right) d x d y .
$$

The proof indicates that if $\nu>0$ or if $x$ is bounded below away from $1 / \sqrt{2}$, then all the Hilbert spaces in this paper can be replaced by $L^{2}$. 


\section{Uniqueness}

In this section we establish a fundamental a priori estimate.

Lemma $3 \forall w \in W \exists K \in \mathbb{R}^{+}{ }_{\ni} K\|w\|_{*} \leq\|L w\|^{*}$.

The constant $K$ depends only on $\varepsilon$ and $\delta$. Lemma 3 implies uniqueness of the adjoint problem for the system (6)-(8). If $v_{1}$ and $v_{2}$ are two solutions, then by linearity, $v_{1}-v_{2}$ lies in the kernel of $L$, and is zero in $W$ by the lemma.

Proof of Lemma 3. We use an abbreviated version of the Friedrichs abc method. Consider the inner product

$$
\begin{gathered}
\left(L w, x y^{2 \kappa} w\right)= \\
\iint_{\Omega}\left\{\left[\left(1-x^{2}\right) w_{1}\right]_{x}-2 x y w_{1 y}+\left[\left(1-y^{2}\right) w_{2}\right]_{y}\right\} x y^{2 \kappa} w_{1} d x d y \\
+\iint_{\Omega}\left(1-y^{2}\right)\left(w_{1 y}-w_{2 x}\right) x y^{2 \kappa} w_{2} d x d y=\iint_{\Omega} \sum_{i=1}^{5} \tau_{i} d x d y
\end{gathered}
$$

where

$$
\begin{gathered}
\tau_{1}=\frac{1}{2}\left[\left(1-x^{2}\right) x y^{2 \kappa} w_{1}^{2}\right]_{x}-x^{2} y^{2 \kappa} w_{1}^{2}-\frac{1}{2}\left(1-x^{2}\right) y^{2 \kappa} w_{1}^{2} \\
\tau_{2}=-\left[x^{2} y^{2 \kappa+1} w_{1}^{2}\right]_{y}+x^{2} y^{2 k} w_{1}^{2}+2 \kappa x^{2} y^{2 k} w_{1}^{2} \\
\tau_{3}=\left[\left(1-y^{2}\right) x y^{2 \kappa} w_{1} w_{2}\right]_{y}-2 \kappa\left(1-y^{2}\right) x y^{2 \kappa-1} w_{1} w_{2}-\left(1-y^{2}\right) x y^{2 \kappa} w_{1 y} w_{2} \\
\tau_{4}=\left(1-y^{2}\right) w_{1 y} x y^{2 \kappa} w_{2} \\
\tau_{5}=-\frac{1}{2}\left[\left(1-y^{2}\right) x y^{2 \kappa} w_{2}^{2}\right]_{x}+\frac{1}{2}\left(1-y^{2}\right) y^{2 \kappa} w_{2}^{2} .
\end{gathered}
$$

Strictly speaking, a limiting argument is required in (17) in case $2 \kappa-1$ is nonpositive. But this case can be ruled out without loss of generality by taking $\mu \geq 2 / 3$.

We ignore for a moment derivatives of products, as these will be integrated and become boundary terms. The coefficients of $w_{1 y} w_{2}$ sum to zero in (17) and (18). After cancellations, the coefficients of $w_{1}^{2}$ in (15) and (16) sum to

$$
\left[-\frac{1}{2}\left(1-x^{2}\right)+2 \kappa x^{2}\right] y^{2 \kappa} \geq\left[-\frac{\mu}{2}+2 \kappa(1-\mu)\right] y^{2 \kappa}
$$




$$
\geq \frac{\varepsilon y^{2 \kappa}}{2} \text {. }
$$

(Notice that if $\nu>0$, then the right-hand side of expression (20) can be bounded below by $\varepsilon \nu^{2 \kappa} / 2>0$.) The coefficient of $w_{2}^{2}$ in (19) is

$$
\frac{1}{2}\left(1-y^{2}\right) y^{2 \kappa} \geq \frac{\delta y^{2 \kappa}}{2}
$$

(Again, this can be bounded below by a positive constant if $\nu>0$.) The coefficient of $w_{1} w_{2}$ in (17) is nonnegative as $x$ and $y$ lie in the fourth quadrant, and we neglect this term in estimating a lower bound for the left-hand side of (14).

Applying Green's Theorem to derivatives of products in (15)-(17) and (19) results in a boundary integral of the form

$$
\begin{gathered}
\int_{\partial \Omega} \frac{x y^{2 \kappa}}{2}\left[\left(1-x^{2}\right) w_{1}^{2}-\left(1-y^{2}\right) w_{2}^{2}\right] d y+ \\
\int_{\partial \Omega} x y^{2 \kappa}\left[x y w_{1}^{2}-\left(1-y^{2}\right) w_{1} w_{2}\right] d x .
\end{gathered}
$$

Condition (9) implies that on $\gamma$,

$$
-\left(1-y^{2}\right) w_{1} w_{2} d x=\left(1-y^{2}\right) w_{2}^{2} d y
$$

so the boundary integral on $\gamma$ reduces to

$$
\begin{gathered}
\int_{x=1} \frac{x y^{2 \kappa}}{2}\left[\left(1-x^{2}\right) w_{1}^{2}+\left(1-y^{2}\right) w_{2}^{2}\right] d y+\int_{x=1} x^{2} y^{2 \kappa+1} w_{1}^{2} d x \\
=\int_{x=1} \frac{x y^{2 \kappa}}{2}\left(1-y^{2}\right) w_{2}^{2} d y \geq 0 .
\end{gathered}
$$

Because $w_{1}$ vanishes on $C$, the remaining boundary integral is of the form

$$
-\int_{C} \frac{x y^{2 \kappa}}{2}\left(1-y^{2}\right) w_{2}^{2} d y,
$$

which is nonnegative under the given orientation by the hypotheses on $\Omega$.

Using (20) and (21), we find that

$$
\left(L w, x y^{2 \kappa} w\right) \geq K^{\prime} \iint_{\Omega} y^{2 \kappa}\left(w_{1}^{2}+w_{2}^{2}\right) d x d y=K^{\prime}\|w\|_{*}^{2},
$$


where

$$
K^{\prime}=\frac{1}{2}(\min \{\varepsilon, \delta\}) .
$$

It remains to estimate $\left(x y^{2 \kappa} w, L w\right)$ from above, which is an application of Young's inequality. The formal argument which follows can be made precise by replacing $\nu$ by $\nu+\alpha$, where $\alpha \in(0,1)$, and eventually allowing $\alpha$ to tend to zero. For any positive constant $\lambda$ we have

$$
\begin{gathered}
\left(x y^{2 \kappa} w, L w\right)=\iint_{\Omega}\left[\frac{(L w)_{1}}{y^{\kappa}}\left(x y^{3 \kappa} w\right)_{1}+\frac{(L w)_{2}}{y^{\kappa}}\left(x y^{3 \kappa} w\right)_{2}\right] d x d y \\
\leq \frac{1}{\lambda}\left\|\frac{L w}{y^{\kappa}}\right\|_{2}^{2}+\lambda\left\|x y^{3 \kappa} w\right\|_{2}^{2} \leq \frac{1}{\lambda}\|L w\|^{* 2}+\lambda\|w\|_{*}^{2},
\end{gathered}
$$

as $\max _{\Omega}\left|x y^{\kappa}\right|^{2}<1$. Choosing $\lambda$ to be sufficiently small relative to $\varepsilon$ and $\delta$, inequalities (22) and (23) imply the assertion of Lemma 3 with $K=$ $\sqrt{\left(K^{\prime}-\lambda\right) \lambda}$

\section{Existence}

Given the a priori estimate of the preceding section, the proof of existence is straightforward. We briefly outline the argument, following $[\mathbf{M}]$.

Lemma 3 implies (employing limiting arguments where necessary) that

$$
\begin{gathered}
|(w, g)|=\left|\left(y^{\kappa} w, y^{-\kappa} g\right)\right| \leq\left\|y^{\kappa} w\right\|_{2}\left\|y^{-\kappa} g\right\|_{2}=\|w\|_{*}\|g\|^{*} \\
\leq K^{-1}\|L w\|^{*}\|g\|^{*} \leq \widetilde{K}(g)\|L w\|^{*} .
\end{gathered}
$$

Thus the functional $\xi$ defined for fixed $g$ and all $w \in W$ by the formula

$$
\xi(L w)=(w, g)
$$

can be extended to a bounded linear functional on $H$. The Riesz Representation Theorem implies that $\forall w \in W$ there is an $h \in H$ for which

$$
\xi(L w)=(L w, h)^{*} .
$$

Defining $u=\left(u_{1}, u_{2}\right)$ so that

$$
u_{1}=-y^{-2 \kappa} h_{1}
$$


and

$$
u_{2}=-y^{-2 \kappa} h_{2}
$$

we have $u \in U$ as $h \in H$; that is,

$$
\iint_{\Omega} y^{2 \kappa}\left(u_{1}^{2}+u_{2}^{2}\right) d x d y=\iint_{\Omega} y^{-2 \kappa}\left(h_{1}^{2}+h_{2}^{2}\right) d x d y<\infty .
$$

We conclude that

$$
\begin{gathered}
(w, g)=\xi(L w)=(L w, h)^{*}= \\
\iint_{\Omega} y^{-2 \kappa}\left[(L w)_{1} h_{1}+(L w)_{2} h_{2}\right] d x d y= \\
-\iint_{\Omega} y^{-2 \kappa}\left[(L w)_{1} y^{2 \kappa} u_{1}+(L w)_{2} y^{2 \kappa} u_{2}\right] d x d y=-(L w, u) .
\end{gathered}
$$

Comparing the extreme left-hand side of this expression with its extreme right-hand side completes the proof of Theorem 2 .

\section{References}

[B1] E. Beltrami, Teoria fondamentale degli spazii di curvatura costante, Annali di Matematica Pura ed Applicata, ser. 2, 2 (1868), 232-255.

[B2] L. Bers, Mathematical Aspects of Subsonic and Transonic Gas Dynamics, Wiley, New York, 1958.

[H] L. K. Hua, in: Proceedings of the 1980 Beijing Symposium on Differential Geometry and Differential Equations, (S. S. Chern and Wu Wen-tsün, eds.), pp. 627-654, Gordon and Breach, New York, 1982.

[M] C. S. Morawetz, A weak solution for a system of equations of elliptichyperbolic type, Commun. Pure Appl. Math. 11 (1958), 315-331.

[VY] O. Veblen and J. W. Young, Projective Geometry, Vol. II, Ginn and Co., Boston, 1918.

[Y] Y. Yamamoto, Existence and uniqueness of a generalized solution for a system of equations of mixed type, Ph.D. Dissertation, Polytechnic University of New York, 1994. 